\title{
Los corticoides transtimpánicos son igualmente efectivos que los orales para la hipoacusia neurosensorial súbita idiopática
}

Oral and intratympanic corticosteroid therapy are equally effective for the treatment of sudden sensorineural hearing loss

Rauch SD y col. JAMA. 2011;305(20):2071-9

\section{Objetivos}

Comparar la efectividad de la corticoterapia por vía oral y por vía transtimpánica en la hipoacusia súbita idiopática (HSI).

Diseño, intervención y medición de resultados principales Ensayo clínico aleatorizado de no inferioridad con un seguimiento de seis meses llevado a cabo en 16 centros de práctica otológica e investigación de Estados Unidos. Fueron reclutados 250 pacientes mayores de 18 años (151 hombres), con HSI unilateral establecida en un período de 72 horas, ocurrida en los 14 días previos. El promedio de umbral de la audiometría tonal (PTA) para las frecuencias 500, 1000, 2000 y $4000 \mathrm{~Hz}$ en el oído afectado debía situarse en $50 \mathrm{~dB}$ o más, con una diferencia de al menos $30 \mathrm{~dB}$ en una de las frecuencias en relación al oído sano.

121 pacientes recibieron $60 \mathrm{mg} /$ día de prednisona por vía oral durante 14 días y cinco días de disminución gradual de la misma $(50 \mathrm{mg} /$ día, $40 \mathrm{mg} / \mathrm{día}, 30 \mathrm{mg} /$ día, $20 \mathrm{mg} / \mathrm{día}$ y 10 $\mathrm{mg} / \mathrm{día}$ ) completando 19 días de tratamiento. 129 pacientes recibieron cuatro dosis de $40 \mathrm{mg} / \mathrm{mL}$ de metilprednisolona cada tres o cuatro días, inyectada en el oído medio a través de la membrana timpánica durante 14 días.

Ambos tratamientos fueron analizados por intención de tratar, a través de una audiometría tonal a los dos y a los seis meses de finalizados, decidiéndose que la diferencia entre ambos tratamientos era clínicamente relevante si era mayor a $10 \mathrm{~dB}$.

\section{Resultados Principales}

En el grupo medicado con prednisona oral se observó un aumento de la PTA de $30,7 \mathrm{~dB}$ a los dos meses y en el tratado con metilprednisolona transtimpánica, de $28,7 \mathrm{~dB}$. La diferencia a favor de la prednisona oral fue de $2,0 \mathrm{~dB}$ con un límite superior del intervalo de confianza $95 \%$ de $6,6 \mathrm{~dB}$. Esta diferencia máxima de 6,6 dB aun estaba por debajo de los $10 \mathrm{~dB}$ requeridos para rechazar la hipótesis de no inferioridad del tratamiento por vía transtimpánica. A los seis meses no hubo diferencias estadísticamente significativas entre los grupos en la PTA y tampoco en el porcentaje de discriminación logoaudiométrica.

\section{Conclusiones}

En pacientes con hipoacusia neurosensorial súbita idiopática el tratamiento con metilprednisolona por vía transtimpánica es igualmente efectivo que el realizado con prednisona por vía oral.

Fuente de financiamiento: National Institute on Deafness and Other Communication Disorders. No se describen conflictos de intereses.

\section{Comentario}

La HSI es la pérdida neurosensorial de por lo menos $30 \mathrm{~dB}$ en tres frecuencias consecutivas, de aparición brusca o durante un período no mayor a 72 horas $^{1}$, que puede acompañarse de vértigo. Clásicamente, su incidencia ha sido reportada entre 5 y 20 casos cada 100.000 habitantes por año ${ }^{2,3}$ aunque recientemente otros autores la han descripto entre 1604 y 300 casos cada 100.000 habitantes por año ${ }^{5,6}$. Además, como $32 \%$ a $65 \%^{7,8}$ de los pacientes se recuperan espontáneamente sin consultar al sistema de salud ${ }^{9}$, se vuelve difícil determinar su incidencia real y evaluar la efectividad en los protocolos de tratamiento.

Una vez realizado el diagnóstico presuntivo de HSI a través de un exhaustivo interrogatorio y un examen clínico otorrinolaringológico tendiente a descartar otras probables causas de pérdida auditiva, se la confirma a través de una audiometría tonal con logoaudiometría ${ }^{10}$. La evaluación se completa con una resonancia magnética de conducto auditivo interno con y sin gadolinio para descartar patología del oído interno y tumores del ángulo pontocerebeloso ${ }^{11}$ y con un análisis de laboratorio para la pesquisa de trastornos autoinmunes sistémicos ${ }^{12}$. Una vez que el diagnóstico es sospechado el paciente debe ser derivado rápidamente al otorrinolaringólogo para tratamiento.

Las opciones de tratamiento incluyen: corticoides orales y transtimpánicos, vasodilatadores, expansores plasmáticos, antivirales, carbógeno, hemodiluyentes, vitaminas y cámara hiperbárica ${ }^{12}$. En estos últimos años algunos autores de importantes centros han reportado buenos resultados con tratamiento con corticoides por vía oral ${ }^{13} \sin$ embargo en una revisión de Cochrane su efectividad no queda clara ${ }^{14}$.

\section{Conclusiones del comentador}

Si bien en la actualidad la administración de altas dosis de corticoides por vía oral es considerada el tratamiento estándar ${ }^{8}$ de la HSI, la efectividad documentada por el estudio de Rauch y col. que hemos resumido abre una nueva opción terapéutica para quienes no puedan recibir corticoides por vía oral.

Gabriela Pérez Raffo [ Servicio de Otorrinolaringología. Hospital Italiano de Buenos Aires, gabriela.perezraffo@ hospitalitaliano.org.ar ]

Pérez Raffo G. Los corticoides transtimpánicos son igualmente efectivos que los orales para la hipoacusia neurosensorial súbita idiopática. Evid. Act. Práct. Ambul. 2012. Abr-Jun. 15(2). 48. Comentado de: Rauch S y col. Oral vs intratympanic corticosteroid therapy for idiopathic sudden sensorineural hearing loss: a randomized trial. JAMA. 2011;305(20):2071-9. PMID: 21610239.

\section{Referencias}

1. National Institute on Deafness and Other Communication Disorders. Sudden Deafness. Bethesda, MD: National Institutes of Health; 2000. Publication no. 00-4757.

3. Byl FM Jr. Sudden hearing loss: eight years' experience and suggested prognostic table. Laryngoscope 1984;94:64-61.

4. Teranishi M y col. Thirty-year trends in sudden deafness from four nationwide epidemiological surveys in Japan. Acta Otolaryngol. 2007;127:1259-65.

5. Klemm E y col. A Present Investigation of the Epidemiology in Idiopathic Sudden Sensorineural Hearing Loss Laryngorhinootologie 2009; 88(8): 524-7.

6. Olzowy B y col. The incidence of sudden hearing loss is greater than previously assumed. MMW Fortschr Med 2005; 147(14): 37-8.

7. Mattox DE, Simmons FB. Natural history of sudden sensorineural hearing loss. Ann Otol Rhinol Laryngol 1977; 86:463-480.

8. Wilson W y col. The efficacy of steroids in the treatment of idiopathic sudden hearing loss: a double-blind clinical study. Arch Otolaryngol. 1980;106(12):772-776.

9. Simmons FB. Sudden idiopathic sensorineural hearing loss: some observations. Laryngoscope. 1973;83(8):1221-1227.

10. O'Malley M y col. Sudden hearing loss. Otolaryngol Clin North Am. 2008:41(3):633-49.

11. Magnetic resonance imaging in sudden deafness. Ramos HV, Barros FA, Yamashita H, Penido Nde O, Souza AC, Yamaoka WY. Braz J Otorhinolaryngol. 2005;71(4):422-6.

12. O'Malley M y col. Sudden hearing loss. Otolaryngol Clin North Am. 2008 Jun;41(3):633-49.

13. Slattery $W$ y col. Oral steroid regimens for idiopathic sudden sensorineural hearing loss. Otolaryngol Head Neck Surg 2005;132:5-10.

14. Wei B y col. Steroids for idiopathic sudden sensorineural hearing loss. Cochrane Database Syst Rev. 2006 Jan 25;(1):CD003998. 